\title{
A Novel System to Detect Forest Land Cover Change
}

\author{
Pratik Kale \\ Department of \\ Information \\ Technology, \\ APCOER, Pune-09
}

\author{
Priya Kale \\ Department of \\ Information \\ Technology, \\ APCOER, Pune-09
}

\author{
Ruksar Kalyani \\ Department of \\ Information \\ Technology, \\ APCOER, Pune-09
}

\author{
Dhanashri Joshi \\ Department of \\ Information \\ Technology, \\ APCOER, Pune-09
}

\begin{abstract}
Land use is forced by environmental factors such as soil characteristics, climate, topography, and vegetation. Image processing helps to identify the type of land, by displaying particular image of that area and that image will be helpful to classify the land in the form of percentage. Existing methodologies do the change detection procedure by detecting the objects in image and that objects are compared with the base image objects to obtain a difference image. This paper a proposed system is used to develop a suitable method related to land areas for finding changes in land areas that undergoes changes over a period of time. In proposed method to get a clear image pre-processing is done. In pre-processing, the methods namely denoising, resizing and control point selection is done. Image segmentation and image classification is done on the image to get the final percentage change in forest land.
\end{abstract}

\section{Keywords}

Image pre-processing, Image segmentation, image classification, canny edge detection and k-NN classifier

\section{INTRODUCTION}

Image processing is a technique to convert an image into digital form sequentially to get an enhanced image or to extract some useful data from it and do some processing on it. It is a type of signal dispensation in which input is image, like video or photograph and output may be image or features associated with that image.

Importance and requirement of digital image processing from two main application areas: the first being the Improvement of pictorial information for human understanding and the second being the Processing of an extract data for an autonomous machine understanding. Digital image processing has a broad range of applications such as remote sensing, image and data storage for transmission in applications like business, medical,imaging, audio imaging, Forensic sciences and industrial automation or robotics. Images acquired by satellites are useful in tracking of earth resources, geographical mapping, and forecast of agricultural crops, urban population, weather forecasting, flood and fire control.

Deforestation is permanent damage of forest in order to make the land available for other use. The mother land have already lost nearly $50 \%$ of the world's original forest, each year the land loses 13 million hectares of forests the equivalent of 36 football fields per minute. The proposed system that will show the changes that occur in land by comparing the previous image and input image. By performing the steps on the input image we will achieve the result in the form of percentage of change in land cover and land use.

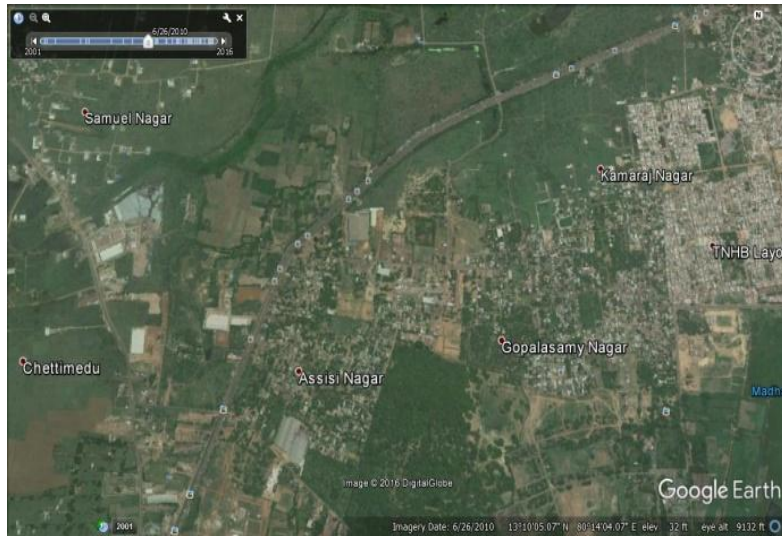

Figure a. Chennai 2010 [10]

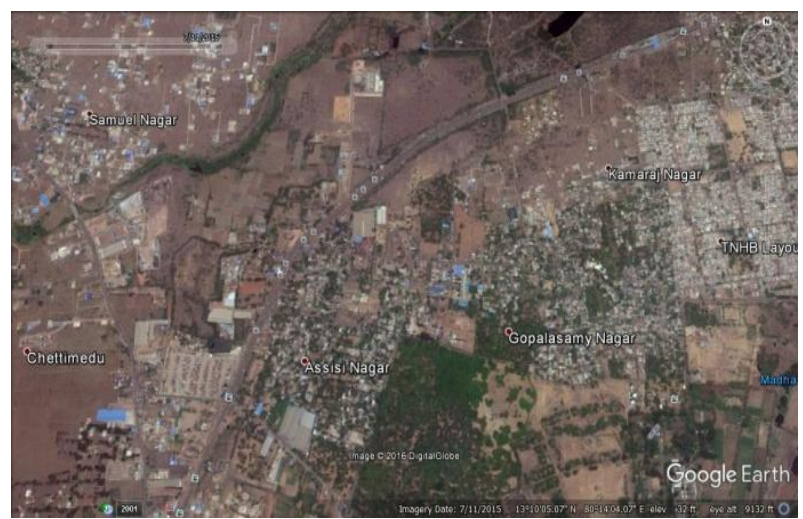

Figure b. Chennai 2015 [10]

As an example, the images of nearby cities of Chennai are taken to show the changes in the land of that particular area. The images are taken from Google Earth in Multi-Temporal type and in .jpeg format. As shown in above figure a and figure $b$ the difference or change in five years in nearby cities of Chennai is shown. A drastic change is occurred in the land in most of the areas. The forest or the greenery is degrading due to the urbanization.

In figure a, the most of the land is covered with forest as compared in the figure $b$. And in figure $b$ due to urbanization or human activities and natural calamities the forest area is degraded.

\section{LITERATURE SURVEY}

This section contains the various techniques and methods described by the authors. The below table 1 will give the overview of list of papers that are related to the proposed system. 
Table 1. Literature Survey

\begin{tabular}{|c|c|c|c|}
\hline Sr.no & Year & Paper name & Description \\
\hline 1. & 2015 & $\begin{array}{l}\text { An Application of } \\
\text { Image Change } \\
\text { Detection-Urbanization }\end{array}$ & $\begin{array}{l}\text { Pre-Processing } \\
\text { operation and } \\
\text { Image } \\
\text { Segmentation }\end{array}$ \\
\hline 2. & 2015 & $\begin{array}{l}\text { Fusion of RADARSAT- } \\
2 \quad \text { Imagery with } \\
\text { LANDSAT-8 } \\
\text { Multispectral Data for } \\
\text { Improving Land Cover } \\
\text { Classification } \\
\text { Performance } \\
\text { SVM Using }\end{array}$ & $\begin{array}{l}\text { RADARSAT-2 } \\
\text { imageWavelet- } \\
\text { based fusion } \\
\text { (WT) techniques }\end{array}$ \\
\hline 3. & 2015 & $\begin{array}{l}\text { Land-Cover Classification } \\
\text { of Remotely Sensed } \\
\text { Images Using Compressive } \\
\text { Sensing Having Severe } \\
\text { Scarcity of Labelled } \\
\text { Patterns }\end{array}$ & $\begin{array}{l}\text { Compressive } \\
\text { sensing (CS) } \\
\text { approach domain } \\
\text { adaptation (DA) }\end{array}$ \\
\hline 4. & 2014 & $\begin{array}{l}\text { Parcel-Based Change } \\
\text { Detection in Land-Use } \\
\text { Maps by Adopting the } \\
\text { Holistic Feature }\end{array}$ & Spatial Envelope \\
\hline 5. & 2013 & $\begin{array}{l}\text { Hybrid classification of } \\
\text { Landsat data for land } \\
\text { cover changes analysis } \\
\text { of the Halabja city, Iraq }\end{array}$ & $\begin{array}{l}\text { Hybrid } \\
\text { classification,Pos } \\
\text { t classification }\end{array}$ \\
\hline 6. & 2012 & $\begin{array}{l}\text { Image Segmentation } \\
\text { Techniques }\end{array}$ & $\begin{array}{l}\text { Segmentation } \\
\text { Ultrasound images, } \\
\text { Synthetic Aperture } \\
\text { Radar(SAR) images }\end{array}$ \\
\hline 7. & 2012 & 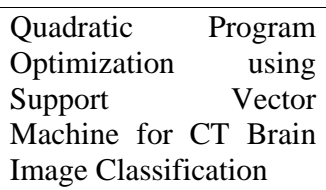 & SVM Technique \\
\hline
\end{tabular}

\section{A.Classification method}

Jovit Reno. A, Beulah David. D [1] has proposed an efficient method for finding changes in land areas that undergoes changes over period of time. They have used multi-temporal data as input image and these images are collected from Google earth. The system uses many pre-processing steps to obtain a clear an efficient pre-processed image. Image segmentation is used to separate the useful pixels in the image. The image segmentation is done using Canny edge detection method for identifying the edges in the image. For image classification, Feed forwarding back propagation is used. The output is shown by calculating performance analysis and Roc (Receiver operating curve) of the total performance of the change detection.

Moumita Roy, FaridMelgani, Ashish Ghosh, Enrico Blanzieri, and Susmita Ghosh, [2] they have used multispectral remote sensing images and then firstly they have assess the compressive sensing (CS) approach as a classification tool for those image type. And then they have proposed a new strategy of using CS approach to perform domain adaptation for classifying images at large spatial scales like continental mapping. They have also used domain adaptation technique.

ChanikaSukawattanavijit, Jie CHEN, [3] has used the SVM ( Support Vector Machine) classifier to improve the land cover classification for utility multi-source RADARSAT-2 and LANDSAT-8 multi-spectral images. The WT (Wavelet-based fusion) techniques are implemented in the data fusion process. The RBF (Radical Basic Function) kernel functions were used with SVM classifier to classify land cover types. The results of the Support Vector Machine classification are matched with ML (Maximum Likelihood) classifier. The land cover classification includes steps such as i) image processing, ii) image fusion, iii) support vector machine, iv) accuracy. The main motto of this paper was to improve land cover types classification using Support Vector Machine classifier to various datasets.

Jwan Al-doski, ShattriB.Mansor and Helmi ZulhaidiMohdShafrihave applied the remote sensing technology for monitoring land cover changes. Their study was conducted to observe the impacts of wars on land cover in the Halabja city in the northern part of Iraq (study area).They used Land sat $5 \mathrm{TM}$ and Land sat 7 ETM+ data acquired in 990 and 2000 were used. A post-classification technique base on hybrid classification was taken. After performing pre- processing step an unsupervised K-means classification was carried out firstly on six reflective bands with the support of supplementary data for two images independently followed by maximum likelihood supervised classification to classify all images into five land cover classes; water bodies, vegetation fields, forests, built-up or urban areas and bare or plain lands. The images were collected from Google earth images. They performed Kmeans classification, MLC (Maximum Likelihood classification) and Hybrid classification on two images. Changes on images were observed across all five land cover classes: Water bodies, Forests, Vegetation Fields, Built-up or Urban Areas and Bare lands [5].

J Umamaheswari and Dr.G.Radhamanihas used an effective Computer Tomography (CT) image classification using Support Vector Machine (SVM) with optimized quadratic programming methodology is estimated. Due to manual understanding of brain or mind images based on visual examination by radiologist/physician that cause inappropriate diagnosis, when a large number of CT images are examine. To avoid the human error, an automated optimized classification system is proposed for abnormal Computer Tomography (CT) image identification. According to the author this is computerized system for content based image retrieval with better classifier accuracy and prediction time. SVM classifier can correctly sequence up the data's as normal and abnormal brains understood manually by the user. Thus the system can retrieve more number of images present in the query database. The proposed classifier is analysed with existing Sequential Minimal Optimization (SMO) and K Nearest Neighbor classifier $(\mathrm{KNN})$. The image feature is extracted using MATLAB for both normal and abnormal [7].

\section{B. Spatial envelope}

Wu Bin, Yang Jian, Zhao Zhongming, Meng Yu, YueAnzhi, Chen Jingbo, He Dongxu, Liu Xingchun, and Liu Shunxi[4] have introduced the method of parcel based changed detection by using holistic feature called spatial envelope i.e. without segmenting it into identical objects or small regions it encodes each parcel. Holistic means characterized by the conviction that the parts of something are interconnected and understandable only by reference to the 
whole. The land-use parcels are clipped by polygons in the land-use map with the energy spectrum of WFT. WFT is nothing but Windowed Fourier Transform. It performs the WFT of each land use parcel. In this paper they have calculated spatial feature, spectral feature, Textural feature, gradient feature. Their first dataset is for a region of southern china in the year 2011 and 2012. And the second dataset is for the region Beijing. And the third dataset which aims at validating that this method is effective with image from different sensors. Therefore according to them parcel-based analysis is more suitable in land-use management and applications.

\section{Image Segmentation Technique}

Rajesh warDass, Priyanka, Swapna Devi [6] has described that based on different skills image segmentation methods are at present divided into following categories, based on two assets : i] Detecting Discontinuities which means to partition an image based on sudden changes in intensity and it includes edge detection algorithm. ii] Detecting Similarities which means to divide an image into regions or areas that are same according to a set of predefined condition. It includes algorithms like thresholding, region growing, region splitting and merging.

The authors have also explained about many image segmentation techniques which are as follows:- A] Segmentation Based on Edge Detection, B] Thresholding method, C] Region Based Segmentation Method, D] Segmentation Methods Based on PDE ( Partial Differential Equation), E] Segmentation Based on Artificial Neural Network, F] Segmentation Based on Clustering, G] Multiobjective Image Segmentation.

\section{PROPOSED SYSTEM}

The proposed system contains the steps such as pre-processing, image segmentation, and image classification on image. The layout of this work is given in Figure. 1. Each step is elaborated in detail. Canny edge detector and $\mathrm{kNN}$ algorithm as described below.

\section{A. Pre-processing}

The most important step in image processing is pre-processing. Technically to achieve the clear image pre-processing is done. Generally an image contains unwanted particles which are not necessary. Unwanted particles means noise i.e. the image is not clear they are blur. Therefore to obtain clear image preprocessing step is done. In this work, three pre-processing steps are performed. Those are denoising, resizing, control point selection.

Denoising: - Digital images are often contaminated by noise during the gaining. Image denoising aims at reducing the noise while retaining the image content. For denoising, the NLM (Non-Local Means) method will be used by the proposed system. Resizing is done after the denoising step as shown in fig 1.

Resizing: - Resizing means to adjust the image. Each and every image is of different size. The images are taken from in different directions or sources. The size and the direction of the images are depending upon the distance and the direction of the satellite. Therefore to get the equal size of the image resizing operation is used.

Control point selection is used to select the particular area or region for the process. The control point selection method will be called by the application in proposed system. After selecting a particular region or area the image or output after the control point selection method is stored in database and then it will be further send to image segmentation process for segmenting.

\section{B. Image Segmentation}

As shown in the figure 1, after pre-processing, the image segmentation has to be done. Image segmentation is the process of partitioning an image into many segments, so as to change the representation of an image into some understandable form that is more meaningful and easier to analyze. The purpose of edge detection in general is to significant reduce the sum of data in an image, while preserving the structural properties to be used for further image processing. There are no optimal algorithms for image segmentation.

In this work, canny edge detection method is used for edge detection because even though it is quite old, it has become one of the standard edge detection method and it is still used in study till date.

\section{Canny edge Detector}

The algorithm runs in 5 steps:

1. Smoothing: Using Gaussian operator to the image will be blurred and then the unwanted noise from that blur image will be removed. And then image will be sent to next step for processing.

2. Finding gradients: The edges should be marked or clear where the gradients of the image have large magnitudes.

3. Non-maximum suppression: local maxima should be marked as edges or end of the image

4. Double thresholding: In this step, potential edges are determined by thresholding.

5. Edge tracking by hysteresis Final edges are determined by destroying all edges that are not connected to a very certain (strong) edge.

\section{Image classification}

After image segmentation our next step is image classification. As the name suggests image classification means to classify the image. Here, we will classify the image with the previous resultant image and compare it. The RGB values will be compared and then the image will be classified. After these step the image will go to the analysis state in which again the resultant image after image classification will be analysed or studied and then final output will be given. The KNN classification algorithm will be used to classify the images.

\section{KNN (K Nearest Neighbor) algorithm is as follows:-}

- The k-Nearest Neighbors algorithm is a most popular method used for classification and regression.

- In k-NN classification, an object or a thing is classified by a majority vote of its neighbors and the output is a class membership. The object being assigned to the class most common among its $\mathrm{k}$ nearest neighbors $(\mathrm{k}$ is a positive integer, typically small). If $\mathrm{k}=3$, then the object is simply assigned to the class of that three nearest neighbor.

- In k-NN regression or classification, the output is the property value for the object as explain in above paragraph. This value is the standard or average of the values of its $\mathrm{k}$ nearest neighbors. 


\section{BLOCK DIAGRAM OF PROPOSED SYSTEM}

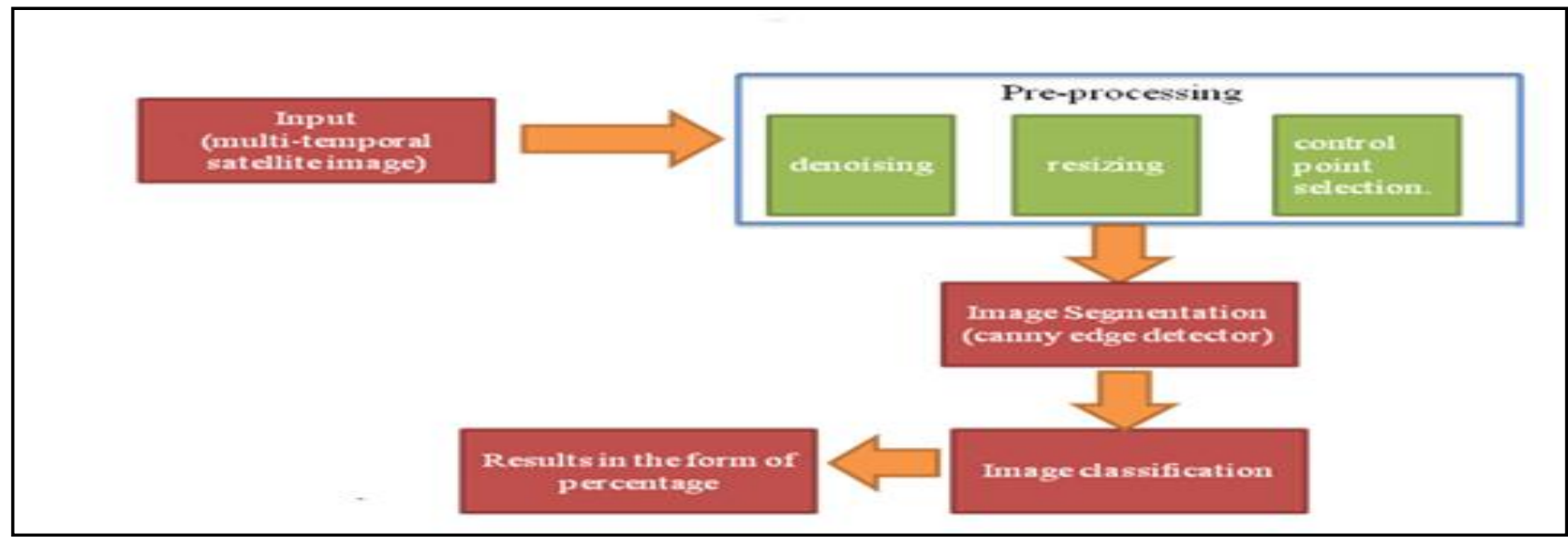

Figure 1.Block Diagram

\section{ACKNOWLEDGMENTS}

I am extremely thankful to my Project guide Prof. D. P. Joshi for suggesting the topic for dissertation and providing all the assistance needed to complete the work. She inspired me greatly to work in this area. Her willingness to motivate me contributed tremendously to my project. Her guidance and discussions are invaluable in realization of this report.

\section{CONCLUSION}

By analyzing and studying the existing method the proposed system will give the difference between the previous image and the current image of a land. Canny edge detector is the most present method to detect the edges in the image and the edge detected image will go for the classification using kNN classifier algorithm, the system will discover the change in the image in the form of percent change in the forest land area.

The Proposed system can be extended to the government level including the factors such as urban areas, vegetation fields, bare lands and weather forecasting and also for water bodies etc. ThusAndroid and Web Application can also be developed in future to give results anytime and anywhere.

\section{REFERENCES}

[1] Jovit Reno. A, Beulah David. D , "An Application of Image Change Detection- Urbanization", 2015 International Conference on Circuit, Power and Computing Technologies [ICCPCT] .

[2] Moumita Roy, Farid Melgani, Senior Member, IEEE, Ashish Ghosh, Member, IEEE, Enrico Blanzieri, Member, IEEE, and Susmita Ghosh, Member, IEEE, “ Land-Cover Classification of Remotely Sensed Images Using Compressive Sensing Having Severe Scarcity of Labeled Patterns ", IEEE GEOSCIENCE AND REMOTE SENSING LETTERS, VOL. 12, NO. 6, JUNE 2015.

[3] Chanika Sukawattanavijit, Jie CHEN, member IEEE, “ Fusion of RADARSAT-2 Imagery with LANDSAT-8 Multispectral Data for Improving Land Cover Classification Performance Using SVM ",2015 IEEE 5th Asia-Pacific Conference on Synthetic Aperture Radar(APSAR).
[4] Wu Bin, Yang Jian, Zhao Zhongming, Meng Yu, Yue Anzhi, Chen Jingbo, He Dongxu, Liu Xingchun, and Liu Shunxi, "Parcel-Based Change Detection in Land-Use Maps by Adopting the Holistic Feature", IEEE JOURNAL OF SELECTED TOPICS IN APPLIED EARTH OBSERVATIONS AND REMOTE SENSING, VOL. 7, NO. 8, AUGUST 2014.

[5] Jwan Al-doski, Shattri B.Mansor* and Helmi Zulhaidi Mohd Shafri, "Hybrid classification of Landsat data for land cover Changes analysis of the Halabja city, Iraq", 2013 Fifth International Conference on Geo-Information Technologies for Natural Disaster Management.

[6] Rajeshwar Dass, Priyanka, Swapna Devi , "Image Segmentation Techniques", IJECT Vol. 3, Issue 1, Jan. March 2012.

[7] J Umamaheswari and Dr.G.Radhamani, "Quadratic Program Optimization using Support Vector Machine for CT Brain Image Classification", IJCSI International Journal of Computer Science Issues, Vol. 9, Issue 4, No 1, July 2012.

[8] https://www.google.co.in/search?client=ubuntu\&channel $=$ fs $\& q=+$ deforestration + images $\&$ ie $=$ utf $-8 \& o e=u t f$ 8\&gfe_rd=cr\&ei=LPzkV6XPM7L98wejzIygCw\#channe $\mathrm{l}=\mathrm{fs} \& \mathrm{q}=$ deforestration +

[9] https://www.google.co.in/search?client=ubuntu\&channel $=\mathrm{fs} \& \mathrm{q}=+$ deforestration+images $\&$ ie $=$ utf $-8 \&$ oe $=u t f$ 8\&gfe_rd=cr\&ei=LPzkV6XPM7L98wejzIygCw\#channe $\mathrm{l}=\mathrm{fs} \& \mathrm{q}=$ canny+edge+detection+algorithm

[10] https://www.google.co.in/?gfe_rd=cr\&ei=Vf_kV6aM4zT 8gfxo73IAw\&gws_rd=ssl\#q=google+earth+images

[11] https://www.analyticsvidhya.com/blog/2014/10/introduct ion-k-neighbours-algorithm-clustering

[12] https://www.google.co.in/?gfe_rd=cr\&ei=Vf_kV6aM4zT8gfxo73IAw\&gws_rd=ssl\#q=image+preprocessi ng 